\title{
Predictors for outcomes and readmission rates following double balloon enteroscopy: a tertiary care experience
}

\section{()(1) $\odot$}

\section{Authors}

Danielle Jarrard Shelnut ${ }^{1}$, Omar T. Sims ${ }^{2}$, Jenine N. Zaibaq ${ }^{1}$, Hyejung Oh $^{3}$, Krishna V. Venkata ${ }^{4}$, Shajan Peter ${ }^{5}$

Institutions

1 University of Alabama at Birmingham, Tinsley Harrison Internal Medicine Residency, Birmingham, Alabama

2 University of Alabama at Birmingham, Department of Social Work, College of Arts and Sciences/Department of Health Behavior, School of Public Health/Center for AIDS Research/Comprehensive Center for Healthy Aging, Birmingham, Alabama

3 Troy University, Dothan Campus Ringgold Standard Institution, Department of Human Services \& Social Work, College of Health and Human Services, Dothan, Alabama

4 University of Alabama, Internal Medicine Residency, Montgomery, Alabama

5 University of Alabama at Birmingham, Division of Gastroenterology and Hepatology, Birmingham, Alabama

submitted 17.7.2017

accepted after revision 7.3.2018

Bibliography

DOI https://doi.org/10.1055/a-0602-3967 |

Endoscopy International Open 2018; 06: E751-E757

(c) Georg Thieme Verlag KG Stuttgart · New York

ISSN 2364-3722

Corresponding author

Danielle Jarrard Shelnut, MD, University of Alabama at Birmingham, Tinsley Harrison Internal Medicine Residency, Department of Gastroenterology and Hepatology, 1720 2nd Ave South BDB 321, Birmingham Alabama 352940012, United States

Fax: 205-975-9777

dshelnut@uabmc.edu

\section{ABSTRACT}

Aim The objectives of this study are to examine clinical characteristics of patients undergoing anterograde and retrograde double balloon enteroscopy (DBE) and to assess factors predicting positive diagnostic yield, therapeutic yield, and readmission.

Methods We conducted a retrospective cohort study of patients $(n=420)$ who underwent DBE at a tertiary care center between 2012 and 2016 at a tertiary referral center. Measures of central tendency and frequency distributions were used for univariate analysis. Chi-square and t-test analyses were used to compare patient characteristics. Logistic regression was used to predict outcomes of interest.

Results Of patients included in the study, $59 \%$ were male with a mean age of $61.49(S D=15.15)$ Altered anatomy was noted in $14 \%$, while $5 \%$ and $13 \%$ of patients had end stage renal disease (ESRD) and current use of anticoagulation, respectively. The most common indication for DBE was obscure gastrointestinal bleed (OGIB) (33\%). Fortynine patients had obscure and overt gastrointestinal bleeding (GIB) and $22 \%$ had occult GIB with iron deficiency. The cohort's rate of positive diagnostic yield was $73 \%$ and $35 \%$ for therapeutic yield. The 30-day and 6-month readmission rates were both $11 \%$. A higher proportion of those readmitted were male ( $75 \%$ vs $57 \%, P=0.027$ ) and had longer procedural time ( 38.68 vs $46.57, P=0.011$ ). Likewise, occult GIB with iron deficiency anemia and iron deficiency alone $(\mathrm{OR}=2.45, \mathrm{Cl}: 1.233-4.859, P=0.011)$, inpatient status (OR 2.42, Cl 1.344-4.346, $P=0.003$ ), and longer procedural time $(\mathrm{OR}=1.02, \mathrm{Cl}: 1.004-1.029, P=0.008)$ were associated positively with readmission.

There were no statistically significant predictors of positive diagnostic yield, however procedural time $(\mathrm{OR}=1.01, \mathrm{Cl}$ : 1.03-1.026; $P=.0017)$ and older age $(\mathrm{OR}=1.03, \mathrm{Cl}$ : $1.009-1.045, P=0.003)$ were positively associated with therapeutic yield. Retrograde procedure $(O R=0.230, \mathrm{Cl}$ $0.125-0.422, P=0.000$ ) was negatively associated with therapeutic yield.

Conclusion DBE procedures have relevant efficacy for both diagnostic and therapeutic yield while evaluating small bowel disease. Readmission rates are low and more in those with GI bleed and iron deficiency with longer index procedural times. 


\section{Introduction}

The indications for enteroscopy have continued to expand. Among these include evaluation for iron deficiency anemia, inflammatory bowel disease, and small bowel masses [1,2]. Enteroscopy also allows for therapeutic procedures such as endoscopic retrograde cholangiopancreatography (ERCP), jejunal tube placement, and investigating surgically altered anatomy [3-5]. So far, evaluation for suspected small bowel bleeding is the most common indication despite negative endoscopic evaluation [2]. Current guidelines reserve the term obscure gastrointestinal (GI) bleeding (OGIB) to identify patients not found to have a source of bleeding after performance of standard upper and lower endoscopic examinations, small bowel evaluation with video capsule endoscopy (VCE), or radiographic testing $[6,7]$.

The diagnostic workup of such OGIB can be extensive and require longer hospital stays, blood transfusions, and multiple investigative procedures $[8,9]$. In a prospective study of 60 patients, Kaffes et al. found significant reductions in transfusion requirements and re-bleeding events after interventional double-balloon enteroscopy (DBE) at $10 \pm 5.2$ mo postprocedure [10]. Thus, identifying factors early during admission that are associated with higher risk of re-bleeding or readmission would be advantageous from both a resource utilization and clinical outcomes perspective. A number of retrospective cohort studies have explored various aspects of DBE's performance and utility $[11,12]$. However, the literature lacks evidence in identifying useful predictors of DBE success and outcomes, especially with regards to readmission rates after initial endoscopic evaluation. This study aims to characterize patient and procedural variables that impact both DBE diagnostic and therapeutic yield for various indications. Additionally, we explore whether certain patient characteristics can predict readmission rates.

\section{Methods}

\section{Data collection and study design}

We conducted a retrospective chart review of patients who underwent DBE at the University of Alabama at Birmingham, an academic tertiary center, between November 2012 and August 2016. We excluded patients aged $<18$ years and pregnant patients. The study was approved by the institutional review board. All procedures were performed under monitored anesthesia care using the diagnostic Fujinon Double-Balloon Enteroscopy System (Fujinon Inc., Saitama, Japan) with a 2.2-mm endoscopy accessory channel. Sedation was administered by trained anesthesia personnel for ambulatory care. Routinely, the preferred anesthesia for anterograde procedures was intubation and general anesthesia, while propofol sedation was predominately used for retrograde procedures. Procedures were performed by expert gastroenterologists with extensive experience in interpreting VCE and in performing DBE.

Demographic, clinical, and endoscopic data were extracted during chart review. Indications for the DBE procedures were noted. Obscure GI bleeding or suspected small bowel bleeding, either overt or occult, were classified per recent guidelines [6].
Outcomes of interest were rates and predictors of positive diagnostic yield, therapeutic yield, and readmission. Differences among these outcomes with respect to anterograde or retrograde DBE procedures were examined as well. Positive diagnostic yield was defined as a significant endoscopic finding on DBE that was considered to be clinically relevant to the indication for endoscopy. Therapeutic yield was defined as an endoscopic intervention. Readmission rates were calculated as those who were admitted for a GI-related cause such as GI bleeding overt or occult with iron deficiency. Early readmission was defined as hospital readmission as bedded outpatient or inpatient $30 \mathrm{~d}$ or 6 mo status post initial DBE.

\section{Statistical analysis}

Measures of central tendency and frequency distributions were calculated. Independent t-tests and chi-squared statistics were used for bivariate comparisons between anterograde and retrograde procedures. Logistic regression was used to predict positive diagnostic yield, therapeutic yield, and readmission. All analyses were conducted using SPSS version 22.0 statistical software and statistical significance was set at $P<0.05$.

\section{Results}

Of all patients $(n=420)$ included in the study, $59 \%$ were male with a mean age of 61.49 years (standard deviation [SD] = 15.15 ), and $72 \%$ of procedures were performed in outpatient settings ( $\triangleright$ Table 1a). Thirty-three percent of patients had a body mass index (BMI) $\geq 30$ and $88 \%$ had ASA III/IV classifications. Fourteen percent of patients had altered anatomy, while $5 \%$ and $13 \%$ had end-stage renal disease (ESRD) and current use of anticoagulation (vitamin $\mathrm{K}$ inhibitor, factor $\mathrm{Xa}$ inhibitor, direct thrombin inhibitor, or antiplatelet medication), respectively. Of those patients evaluated for suspected small bowel bleeding, 207 (49\%) had overt and 93 (22\%) had occult GI bleeding. The mean procedural time was $39.58 \mathrm{~min}(\mathrm{SD}=$ 20.08 ), and $74 \%$ of DBE patients received anterograde procedures. In terms of retrograde procedures, cecal intubation and ileal examination was successful in $91.7 \%$ of patients. Reasons for incomplete examination were incomplete preparation, adhesions, and strictures. Total enteroscopy was achieved in $3 \mathrm{pa}-$ tients only, as the strategy was to target and treat the findings or lesion either by anterograde or retrograde approach. This was thought to be a reason for not pursuing total enteroscopy in most of the patients. Tattooing of the distal site of intubation was performed routinely in all included patients.

Compared to retrograde procedures, a higher proportion of DBE patients with anterograde procedures had altered anatomy ( $18 \%$ vs. $4 \%, P=0.000$ ), positive diagnostic yield ( $77 \%$ vs. $62 \%$, $P=0.003$ ), and therapeutic yield ( $41 \%$ vs. $16 \%, P=0.000)(\triangleright \mathrm{Ta}-$ ble 1b). Compared to anterograde procedures, a higher proportion of DBE patients with retrograde procedures were on anticoagulation ( $20 \%$ vs. $11 \%, P=0.010)$ and received MAC anesthesia ( $34 \%$ vs. $8 \%, P=0.000$ ). There were no significant differences in procedural times between anterograde and retrograde procedures ( 38.84 vs. $41.73, P=0.202$ ). The most common kind of altered anatomy was Roux-en-Y gastric bypass. 
- Table 1a Characteristics of DBE patients.

\begin{tabular}{|c|c|}
\hline Variables (categories) & n (\%)/mean (SD) \\
\hline \multicolumn{2}{|l|}{ Sex } \\
\hline - Male & $249(59 \%)$ \\
\hline - Female & $171(41 \%)$ \\
\hline Age, range: $16-93$, mean (SD) & $61.49(15.15)$ \\
\hline \multicolumn{2}{|l|}{ Patient type } \\
\hline - Outpatient & $303(72 \%)$ \\
\hline - Inpatient & $117(28 \%)$ \\
\hline \multicolumn{2}{|l|}{ BMI (rank) } \\
\hline . $<18.5$ & $17(4 \%)$ \\
\hline . $18.5-24.9$ & $126(30 \%)$ \\
\hline . $25-29.5$ & $119(28 \%)$ \\
\hline . $30+$ & $153(36 \%)$ \\
\hline " n/a & $5(1 \%)$ \\
\hline \multicolumn{2}{|l|}{ ASA class (rank) } \\
\hline .1 & $1(0 \%)$ \\
\hline .11 & $49(12 \%)$ \\
\hline . III & $321(77 \%)$ \\
\hline . IV & $47(11 \%)$ \\
\hline \multicolumn{2}{|l|}{ Altered anatomy } \\
\hline - No & $361(86 \%)$ \\
\hline - Yes ${ }^{1}$ & $59(14 \%)$ \\
\hline \multicolumn{2}{|l|}{ Anticoagulation } \\
\hline . No & $363(87 \%)$ \\
\hline - Yes & $55(13 \%)$ \\
\hline \multicolumn{2}{|l|}{ ESRD } \\
\hline - No & $398(95 \%)$ \\
\hline - Yes & $22(5 \%)$ \\
\hline \multicolumn{2}{|l|}{ Anesthesia type } \\
\hline - General & $359(86 \%)$ \\
\hline - MAC (monitored anesthesia care) & $61(15 \%)$ \\
\hline \multicolumn{2}{|l|}{ Pill capsule } \\
\hline . No & $212(51 \%)$ \\
\hline - Yes & $207(49 \%)$ \\
\hline \multicolumn{2}{|l|}{ DBE type } \\
\hline - Anterograde & $311(74 \%)$ \\
\hline - Retrograde & $109(26 \%)$ \\
\hline $\begin{array}{l}\text { Total procedural time, range } 1 \text { - } 149 \text {, } \\
\text { mean (SD) }\end{array}$ & $39.58(20.08)$ \\
\hline \multicolumn{2}{|l|}{ Indications } \\
\hline - Obscure/overt GIB & $207(49 \%)$ \\
\hline
\end{tabular}

- Table 1a (Continuation)

\section{Variables (categories)}

n (\%)/mean (SD)

- Occult GIB w/ iron deficiency and $93(22 \%)$ iron deficiency alone

- Others $^{2}$

$120(29 \%)$

Positive diagnostic yield

- No $112(27 \%)$

- Yes $308(73 \%)$

Types of findings

- Normal

$112(21 \%)$

- Angioectasia/AVM $104(20 \%)$

- Fresh blood, erosions $53(10 \%)$

- Abnormal mucosa $34(6 \%)$

- Others $^{3}$ $230(43 \%)$

Therapeutic yield

- No $275(65 \%)$

- Yes $145(35 \%)$

Types of interventions

- Nothing $275(65 \%)$

- APC $104(22 \%)$

- Epinephrine injection $24(5 \%)$

- Others $^{4}$ $64(14 \%)$

30-d readmission following initial DBE intervention

- No $373(89 \%)$

- Yes $47(11 \%)$

Reason: 30-d readmission

- Non-GI-related reason

- GI-related reason $42(89 \%)$

6-mo readmission following initial DBE intervention

- No $372(89 \%)$

- Yes $48(11 \%)$

Reason: 6-mo readmission

- Non-Gl-related reason $8(17 \%)$

- Gl-related reason $40(83 \%)$

${ }^{1}$ Roux-en-Y gastric bypass (29\%), Billroth (17\%), post-orthotopic liver transplant (OLT) with hepatico-jejunostomy ( $14 \%$ ), others ( $24 \%)$ : Whipple procedure, esophago-jejunostomy, partial gastrectomy-jejunostomy, hemigastrectomy, ileo-sigmoid anastomosis, hepatico-jejunostomy with Roux-en-Y, sleeve gastrectomy, post-OLT with 2 RRCP, abnormal computed tomography (CT) inflammatory bowel diseases. 2 ERCP, abnormal computed tomography (CT), inflammatory bowel disease, polyps, weight loss, incomplete colonoscopy, small bowel obstruction, bile leak/stent exchange, G/J tube malfunction/removal, small bowel ulcers/lesions/mass, stricture, retained VCE capsule, nausea/vomiting, endoscopic feeding tube placement, surveil-

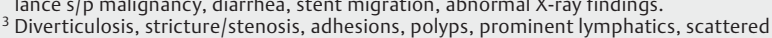
${ }^{3}$ Diverticulosis, stricture/stenosis, adhesions, polyps, prominent lymphatics, scattered
ulcers, healing ulcers, ulcer at small bowel anastomosis, CBD dilation/CBD stones/bile leak, marginal ulcer, Dieulafoy lesion, varices, nodules, small bowel dilation, small bowel mass, retained capsule, external compression, Meckel diverticulum poor prep, bowel mass, retained capsule, external compression, Meckel diverticulum poor prep,
small bowel obstruction, fistulas, mucosal ring, dislodged stent, poor study/food debris.

${ }^{4}$ Hemoclipping, stent removal/exchange/placement, balloon dilation, jejunostomy tube placement/removal, polypectomy, multipolar electrocoagulation, banding, sphincterotomy, fistula closure, endoscopic mucosal resection. 
- Table 1b Bivariate comparison of clinical characteristics between patients with anterograde and retrograde DBE procedures.

\begin{tabular}{|c|c|c|c|}
\hline & Anterograde & Retrograde & $P$-value \\
\hline Sex & & & 0.051 \\
\hline - Male & $193(62 \%)$ & $56(51 \%)$ & \\
\hline - Female & $118(38 \%)$ & $53(49 \%)$ & \\
\hline $\begin{array}{l}\text { Age, range: } 35 \text { - } \\
93, \text { mean (SD) }\end{array}$ & $61.72(14.89)$ & $60.83(15.90)$ & 0.601 \\
\hline Patient type & & & 0.114 \\
\hline - Outpatient & $218(70 \%)$ & $85(78 \%)$ & \\
\hline - Inpatient & $93(30 \%)$ & $24(22 \%)$ & \\
\hline BMI & & & 0.966 \\
\hline . $<30$ & $193(63 \%)$ & $69(63 \%)$ & \\
\hline - $\geq 30$ & $113(37 \%)$ & $40(37 \%)$ & \\
\hline ASA class (rank) & & & 0.680 \\
\hline$\cdot 1 / 11$ & $35(11 \%)$ & $14(13 \%)$ & \\
\hline - III/IV & $273(89 \%)$ & $95(87 \%)$ & \\
\hline Altered anatomy & & & $0.000^{1}$ \\
\hline - No & $256(82 \%)$ & $105(96 \%)$ & \\
\hline - Yes & $55(18 \%)$ & $4(4 \%)$ & \\
\hline Anticoagulation & & & $0.010^{1}$ \\
\hline - No & 277 (89\%) & $86(80 \%)$ & \\
\hline - Yes & $33(11 \%)$ & $22(20 \%)$ & \\
\hline ESRD & & & 0.723 \\
\hline - No & 294 (95\%) & 104 (95\%) & \\
\hline - Yes & $17(5 \%)$ & $5(5 \%)$ & \\
\hline Anesthesia type & & & $0.000^{1}$ \\
\hline - General & 287 (92\%) & $72(66 \%)$ & \\
\hline - Mac & $24(8 \%)$ & $37(34 \%)$ & \\
\hline Pill capsule & & & 0.416 \\
\hline - No & $161(52 \%)$ & $51(47 \%)$ & \\
\hline - Yes & $150(48 \%)$ & $57(53 \%)$ & \\
\hline $\begin{array}{l}\text { Total procedural } \\
\text { time, mean (SD) }\end{array}$ & $38.84(20.01)$ & $41.73(20.21)$ & 0.202 \\
\hline $\begin{array}{l}\text { Positive } \\
\text { diagnostic yield }\end{array}$ & & & $0.003^{1}$ \\
\hline - No & $71(23 \%)$ & $41(38 \%)$ & \\
\hline - Yes & $240(77 \%)$ & $68(62 \%)$ & \\
\hline Therapeutic yield & & & $0.000^{1}$ \\
\hline - No & $183(59 \%)$ & $92(84 \%)$ & \\
\hline - Yes & $128(41 \%)$ & $17(16 \%)$ & \\
\hline Readmission 30-d & & & 0.433 \\
\hline - No & $273(88 \%)$ & $100(92 \%)$ & \\
\hline
\end{tabular}

Table 1b (Continuation)

\begin{tabular}{|l|c|c|c|}
\hline & Anterograde & Retrograde & P-value \\
\hline - Yes & $36(12 \%)$ & $9(8 \%)$ & \\
\hline Readmission 6-mo & & & 0.873 \\
\hline - No & $275(88 \%)$ & $97(89 \%)$ & \\
\hline - Yes & $36(12 \%)$ & $12(11 \%)$ & \\
\hline P $P<0.05$ & & & \\
\hline
\end{tabular}

The rates of positive diagnostic yield and therapeutic yield were $73 \%$ and $35 \%$, respectively, for the procedure. The most frequent findings were Gl angiodysplasia (GIAD) (20\%), fresh blood erosions (10\%), and diverticulosis (5\%). Fresh blood erosions were defined as erosion of mucosa with evidence of bleeding. The general term "abnormal mucosa" was used to describe erythema, granularity, thickened folds, and/or edematous mucosa. The most frequent interventions were argon plasma coagulation (APC) (22\%) and epinephrine injection (5\%). One patient had a colonic perforation during a retrograde procedure secondary to adhesive disease requiring surgical intervention. Otherwise, there were no reported major complications such as pancreatitis or cardiorespiratory events.

In multivariable analysis, there were no independent significant predictors of positive diagnostic yield (table not shown). However, older age (odds ratio $[O R]=1.03$, confidence interval $[C I]: 1.009-1.045, P=0.003)$ and longer procedural time $(O R=$ $1.01, \mathrm{Cl}: 1.03-1.026, P=0.017$ ) were associated positively with therapeutic yield. Likewise, retrograde procedure $(O R=0.230$, $\mathrm{Cl}: 0.125-0.422, P=0.000)$ was associated negatively with therapeutic yield ( $\vee$ Table 2 ).

The 30 - $d$ and 6-mo readmission rates were both $11 \%$. Readmission rates were calculated as those who were admitted for a Gl-related cause such as Gl bleeding overt or occult with iron deficiency.

Compared to patients without $30-\mathrm{d}$ readmission, a higher proportion of patients with $30-\mathrm{d}$ readmission was male $(75 \%$ vs. $57 \%, P=0.027$ ) and had longer procedural time (38.68 vs. 46.57, $P=0.011$ ). Patients with and without $30-d$ readmission did not differ in any other clinical characteristic. In multivariable analysis, occult GIB with iron deficiency anemia $(O R=$ 2.45, Cl: $1.233-4.859, P=0.011)$, inpatient status $(O R=2.42$, $\mathrm{Cl}: 1.344-4.346, P=0.003)$, and longer procedural time $(\mathrm{OR}=$ $1.02, \mathrm{Cl}: 1.004-1.029, P=0.008)$ were associated positively with readmission ( $\downarrow$ Table 3 ). Mean hemoglobin before initial $\mathrm{DBE}$ and time of readmission was $8.41(\mathrm{SD}=1.8)$ and $9(\mathrm{SD}=$ $2.3)$, respectively.

\section{Discussion}

Our study demonstrates that DBE is an effective means to investigate small bowel lesions that were previously unattainable with traditional endoscopy. This corroborates previously published studies describing effectiveness of DBE. Its primary advantage lies in its ability to examine the small bowel in its en- 
Table 2 Multivariate logistic regression predicting therapeutic yield among DBE patients.

\begin{tabular}{|c|c|c|c|c|}
\hline Variables & Categories & OR & $95 \% \mathrm{Cl}$ & $P$-value \\
\hline \multirow[t]{2}{*}{ Sex } & Male & 1 & & \\
\hline & Female & 1.001 & $0.635-1.578$ & 0.998 \\
\hline Age & & 1.027 & $1.009-1.045$ & $0.003^{1}$ \\
\hline \multirow[t]{2}{*}{ BMI } & $\leq 29$ & 1 & & \\
\hline & $\geq 30$ & 0.876 & $0.550-1.394$ & 0.576 \\
\hline \multirow[t]{2}{*}{ ASA class } & $1 / 11$ & 1 & & \\
\hline & $\mathrm{III/IV}$ & 1.677 & $0.735-3.826$ & 0.219 \\
\hline \multirow[t]{2}{*}{ ESRD } & No & 1 & & \\
\hline & Yes & 0.977 & $0.356-2.680$ & 0.964 \\
\hline \multirow[t]{2}{*}{ Pill capsule } & No & 1 & & \\
\hline & Yes & 1.238 & $0.766-2.002$ & 0.383 \\
\hline \multirow[t]{2}{*}{ Patient type } & Outpatient & 1 & & \\
\hline & Inpatient & 0.993 & $0.592-1.664$ & 0.978 \\
\hline Total surgery minutes & & 1.014 & $1.003-1.026$ & $0.017^{1}$ \\
\hline \multirow[t]{3}{*}{ Indication } & Obscure/overt GIB & 1 & & \\
\hline & Occult GIB w/ iron deficiency and iron deficiency alone & 0.854 & $0.479-1.524$ & 0.593 \\
\hline & Other & 1.401 & $0.778-2.524$ & 0.261 \\
\hline \multirow[t]{2}{*}{ DBE type } & Anterograde & 1 & & \\
\hline & Retrograde & 0.230 & $0.125-0.422$ & $0.000^{1}$ \\
\hline
\end{tabular}

- Table 3 Multivariate logistic regression of predicting readmission among DBE patients.

\begin{tabular}{|c|c|c|c|c|}
\hline Variables & Categories & OR & $95 \% \mathrm{Cl}$ & $P$-values \\
\hline \multirow[t]{2}{*}{ Intervention } & No & 1 & & \\
\hline & Yes & 1.204 & $0.673-2.154$ & 0.531 \\
\hline \multirow[t]{2}{*}{ Finding } & No & 1 & & \\
\hline & Yes & 1.385 & $0.693-2.766$ & 0.356 \\
\hline \multirow[t]{3}{*}{ Indication } & Obscure/overt GIB & 1 & & \\
\hline & $\begin{array}{l}\text { Occult GIB bleed w/ iron deficiency and iron deficiency } \\
\text { alone }\end{array}$ & 2.448 & $1.233-4.859$ & $0.011^{1}$ \\
\hline & Other & 1.381 & $0.729-2.617$ & 0.322 \\
\hline \multirow[t]{2}{*}{ Patient type } & Outpatient & 1 & & \\
\hline & Inpatient & 2.417 & $1.344-4.346$ & $0.003^{1}$ \\
\hline Total surgery minutes & & 1.016 & $1.004-1.029$ & $0.008^{1}$ \\
\hline
\end{tabular}

tirety. With the various therapeutic inventions DBE provides, DBE has become a mainstay in evaluation and treatment of suspected small bowel bleeding, abdominal pain, diarrhea, and in patients with altered surgical anatomy. Our study serves to highlight the utility of DBE from a diagnostic and therapeutic standpoint, as well as to characterize factors that impact success rates. In line with prior studies [5,13-16], the most common indication for DBE in our patient population was overt GIB (49\%). Not surprisingly, the most frequent findings were GIAD and fresh blood erosions ( $20 \%$ and $10 \%$, respectively) and the 
most commonly employed interventions were APC and epinephrine injection.

DBE is known to be labor intensive with prolonged endoscopy times. In a study evaluating outcomes of patients with OGIB, Hussan et al. reported average procedural times between 60 and $90 \mathrm{~min}$ [17]. In our experience, the mean procedural time was $39.58 \mathrm{~min}$. However, unlike other cases reported in the literature [18], our study found that the longer procedural times and older age were associated with higher odds of therapeutic yield. The longer procedural time seemed to account for a positive outcome and correlates to the time taken for diagnosis and for appropriate intervention.

Readmission rates, after endoscopy at $30 \mathrm{~d}$ and 6 mo, were low, both at $11 \%$. Nonetheless, presentation of occult Gl bleeding with iron deficiency anemia, inpatient status, and longer procedural times during the initial enteroscopy increased the odds of readmission. The underlying factors associated with the aforementioned readmission rates are unclear. It is plausible that the cases involved were inherently either more significant in terms of degree or number of bleeding foci, were more technically difficult, had ineffective initial therapy, or had lesions that were missed or inaccessible during initial DBE. Inability to locate the "culprit" lesions during the initial endoscopy could account for the longer operator time required. The duration of enteroscopy can be affected by other factors such as obesity, surgical history, presence of adhesions, and other technical factors, though we did not specifically sub-analyze these [19]. On the other hand, it is important to note that prolonged procedural time was conversely correlated with higher therapeutic yield as previously mentioned.

Surprisingly, overt GI bleed did not predict readmission; this could suggest that the culprit lesions were effectively treated in the first place and therefore recurrence in this group was minimal. Inpatient status seemed to affect readmission and this could be attributed to more overt clinical symptoms warranting admission and thus more significant pathology. These factors are important as to effectively minimize cost as well as provide timely intervention. From a large national database study, weekend admissions for bleeding secondary to GIAD were associated with higher odds of mortality, intensive care unit admissions, higher rates of delayed endoscopic procedures, longer lengths of stay, and higher hospital charges [20]. To our knowledge, there are few published studies examining patient variables impacting readmission rates post-DBE. From a recent meta-analysis, in patients treated for small bowel angioectasias after endoscopic therapy, the pooled re-bleeding rate was $34 \%$ after a mean of $22 \pm 13$ mo [21]. For vascular lesions, age $>65$ years, cardiac valvular lesions, renal disease, and use of anticoagulation increased the odds for readmission and thus differed from the predictors that we found [6,22]. However, our study was not limited to patients with Gl bleeding as the sole indication for DBE. Our study is therefore unique with regards to characterizing patient presentations and procedural modalities that impact short-term and long-term readmission for various indications.

Our study also unearths the contrast between the diagnostic and therapeutic yield of anterograde compared to retrograde endoscopy. While the majority of our patients underwent anterograde endoscopy (74\%), retrograde endoscopy was negatively associated with therapeutic yield. It is well known that retrograde endoscopy proves to be more difficult with failure to intubate the terminal ileum in $21 \%$ of DBE cases [23]. It is frequently used to evaluate lesions in the distal small intestine; however, the anterograde approach enables endoscopists to traverse twice as far into the small bowel [6]. It could be inferred that this technical difference accounts for the discrepancies seen. In our case, anterograde endoscopy had a higher rate of diagnostic yield than retrograde endoscopy. Additionally, we encountered that anterograde DBE was useful in evaluation of patients with altered anatomy, specifically in patients with Roux-en-Y anastomoses (28.8\%).

As stated above, our overall diagnostic yield for anterograde DBE was $77 \%$. Previous reports in the literature indicate DBE diagnostic yield for OGIB ranges from $60-80 \%$ [2]. In a systematic review of 66 published studies, anterograde DBE had a pooled detection rate of $68.1 \%$ of all small bowel disease [14]. In regards to the retrograde approach, the diagnostic yield reportedly ranges from $39 \%$ to $47 \%$ [6], which was less than our overall retrograde DBE of $62 \%$. The average pooled therapeutic rate in published literature is approximately $40 \%$ [24], which is in keeping with our study at $35 \%$. In effect, our proficiency and the utility of DBE prove to be clinically comparable to other similar reports in the literature [19].

There were noteworthy limitations and strengths of our study. Our study was retrospective and conducted at a single tertiary care center, thus limiting the generalizability of findings to smaller community settings. Although the multivariable analysis found several findings of statistical significance, the analysis was unable to determine independent predictors of diagnostic yield. Notwithstanding, our study comprised a relatively large clinical sample of patients undergoing DBE $(n=$ 420). To our knowledge, this was one of the larger cohort studies designed to evaluate the diagnostic and therapeutic yield of DBE to date. There was also incomplete follow-up in some of the cases, as our study did not account for those who were not readmitted. Another limitation of the study was that we did not collect other metrics reflecting degree of GI bleed (i. e., transfusion requirement) to establish a pre- and postprocedural analysis of these variables.

In summary, our study reinforces the diagnostic and therapeutic utility of DBE in a large cohort of patients in evaluation of various small bowel conditions including luminal bleeding, abdominal pain, and iron deficiency anemia. Our study highlights important predictors that are positively and negatively associated with therapeutic yield and readmission. Our results indicate that procedural time and increasing age were predictors of therapeutic yield. Moreover, inpatient status, those with Gl bleeding with iron deficiency, and longer procedural times at index were more likely to have readmission. DBE is clearly an important modality for diagnosis and treatment of small bowel disorders. Despite its capabilities, there are a substantial number of patients who require readmission and further treatment. Identifying factors associated with readmission will aid in understanding and implementing the most cost-ef- 
fective strategy with appropriate resource utilization in the algorithmic approach to managing small bowel disorders. Future studies can help in predicting those patients who require longterm follow-up and thereby prevent the need for further interventions.

\section{Competing interests}

None

\section{References}

[1] Skinner M, Peter S, Wilcox CM et al. Diagnostic and therapeutic utility of double-balloon enteroscopy for obscure GI bleeding in patients with surgically altered upper Gl anatomy. Gastrointest Endosc 2014; 80: $181-186$

[2] Velazquez-Avina J, Beyer R, Diaz-Tobar CP et al. New method of direct percutaneous endoscopic jejunostomy tube placement using balloon-assisted enteroscopy with fluoroscopy. Dig Endosc 2015; 27: $317-322$

[3] Gerson LB, Batenic MA, Newsom SL et al. Long-term outcomes after double-balloon enteroscopy for obscure gastrointestinal bleeding. Clin Gastroenterol Hepatol 2009; 7: 664-669

[4] Moreels TG. Update in enteroscopy: New devices and new indications. Digestive Endosc 2017; 30: 174-181

[5] Law JK. New developments in small bowel enteroscopy. Curr Opin Gastroenterol 2016; 32: 387-391

[6] Gerson LB, Fidler JL, Cave DR et al. ACG Clinical Guideline: Diagnosis and Management of Small Bowel Bleeding. Am J Gastroenterol 2015; 110: 1265 -687; quiz 88

[7] Gerson LB. Small bowel bleeding: updated algorithm and outcomes. Gastrointest Endosc Clin N Am 2017; 27: 171 - 180

[8] Santhakumar C, Liu K. Evaluation and outcomes of patients with obscure gastrointestinal bleeding. World J Gastrointest Pathophysiol 2014; 5: 479-486

[9] Samaha E, Rahmi G, Landi B et al. Long-term outcome of patients treated with double balloon enteroscopy for small bowel vascular lesions. Am J Gastroenterol 2012; 107: 240-246

[10] Kaffes AJ, Siah C, Koo JH. Clinical outcomes after double-balloon enteroscopy in patients with obscure $\mathrm{Gl}$ bleeding and a positive capsule endoscopy. Gastrointest Endosc 2007; 66: 304-309
[11] Shishido T, Oka S, Tanaka S et al. Outcome of patients who have undergone total enteroscopy for obscure gastrointestinal bleeding. World J Gastrointest 2012; 18: 666 -672

[12] Monkemuller K, Weigt J, Treiber G et al. Diagnostic and therapeutic impact of double-balloon enteroscopy. Endoscopy 2006; 38: 67-72

[13] Suzuki T, Matsushima M, Okita I et al. Clinical utility of double-balloon enteroscopy for small intestinal bleeding. Dig Dis Sci 2007; 52: $1914-1918$

[14] Xin L, Liao Z, Jiang YP et al. Indications, detectability, positive findings, total enteroscopy, and complications of diagnostic double-balloon endoscopy: a systematic review of data over the first decade of use. Gastrointest Endosc 2011; 74: 563-570

[15] Jovanovic I, Vormbrock K, Zimmermann L et al. Therapeutic doubleballoon enteroscopy: a binational, three-center experience. Dig Dis 2011; 29: (Suppl. 01): 27-31

[16] Yamamoto H, Kita H, Sunada K et al. Clinical outcomes of double-balloon endoscopy for the diagnosis and treatment of small-intestinal diseases. Clin Gastroenterol Hepatol 2004; 2: 1010 - 1016

[17] Hussan H, Crews NR, Geremakis CM et al. Predictors of double balloon endoscopy outcomes in the evaluation of gastrointestinal bleeding. World J Gastrointest Endosc 2014; 6: 248-253

[18] Yamamoto $\mathrm{H}$, Sekine $\mathrm{Y}$, Sato $\mathrm{Y}$ et al. Total enteroscopy with a nonsurgical steerable double-balloon method. Gastrointest Endosc 2001; 53: $216-220$

[19] Committee AT, Chauhan SS, Manfredi MA et al. Enteroscopy. Gastrointest Endosc 2015; 82: 975-990

[20] Serrao S, Jackson C, Juma D et al. In-hospital weekend outcomes in patients diagnosed with bleeding gastroduodenal angiodysplasia: a population-based study, 2000 to 2011. Gastrointest Endosc 2016; 84: $416-423$

[21] Jackson CS, Gerson LB. Management of gastrointestinal angiodysplastic lesions (GIADs): a systematic review and meta-analysis. Am 」 Gastroenterol 2014; 109: 474-483; quiz 84

[22] Rutgeerts P, Van Gompel F, Geboes K et al. Long term results of treatment of vascular malformations of the gastrointestinal tract by neodymium Yag laser photocoagulation. Gut 1985; 26: 586-593

[23] Sanaka MR, Navaneethan U, Kosuru B et al. Antegrade is more effective than retrograde enteroscopy for evaluation and management of suspected small-bowel disease. Clin Gastroenterol Hepatol 2012; 10: 910-916

[24] Sun B, Rajan E, Cheng S et al. Diagnostic yield and therapeutic impact of double-balloon enteroscopy in a large cohort of patients with obscure gastrointestinal bleeding. Am J Gastroenterol 2006; 101: $2011-2015$ 